\title{
A Method of Calculating Critical Depth of Burial of Explosive Charges to Generate Bulging and Cratering in Rock
}

\author{
Mingyang Wang, ${ }^{1,2}$ Songlin Yue, ${ }^{1}$ Ning Zhang, ${ }^{1,3}$ Kanghua Gao, ${ }^{1}$ and Derong Wang1 \\ ${ }^{1}$ State Key Laboratory for Disaster Prevention \& Mitigation of Explosion \& Impact, PLA University of Science and Technology, \\ Nanjing 210007, China \\ ${ }^{2}$ College of Mechanical Engineering, Nanjing University of Science and Technology, Nanjing 210007, China \\ ${ }^{3}$ College of Science, Nanjing University of Science and Technology, Nanjing 210007, China
}

Correspondence should be addressed to Songlin Yue; yslseu@hotmail.com

Received 5 March 2016; Accepted 21 April 2016

Academic Editor: Mohamed B. Trabia

Copyright (C) 2016 Mingyang Wang et al. This is an open access article distributed under the Creative Commons Attribution License, which permits unrestricted use, distribution, and reproduction in any medium, provided the original work is properly cited.

\begin{abstract}
For underground explosions, a thin to medium thickness layer near the cavity of an explosion can be considered a theoretical shell structure. Detonation products transmit the effective energy of explosives to this shell which can expand thus leading to irreversible deformation of the surrounding medium. Based on mass conservation, incompressible conditions, and boundary conditions, the possible kinematic velocity fields in the plastic zone are established. Based on limit equilibrium theory, this work built equations of material resistance corresponding to different possible kinematic velocity fields. Combined with initial conditions and boundary conditions, equations of motion and material resistance are solved, respectively. It is found that critical depth of burial is positively related to a dimensionless impact factor, which reflects the characteristics of the explosives and the surrounding medium. Finally, an example is given, which suggests that this method is capable of calculating the critical depth of burial and the calculated results are consistent with empirical results.
\end{abstract}

\section{Introduction}

Underground explosions have been studied for different purposes [1]. They can be classified into contained explosions (or camouflet explosions), bulging explosions, and cratering explosions. Contained explosions occur when the depth of burial of the explosive is sufficiently deep [2] and the model best suited to such conditions is an explosion in an infinite medium [3]. Bulging explosions occur when the depth of burial of the explosive is within a certain range [4] and are best modelled as explosions in a semi-infinite medium. Cratering explosions may occur in various ways when the depth of burial of the explosive is relatively shallow and they are best modelled as explosions in a semi-infinite medium [5]. A widely accepted zonal model divides the medium near the cavity into a grinding zone, a radial cracking zone, and an elastic zone [6], which can be the basis for the study of bulging and cratering explosions. Owing to their significant military and engineering application, bulging and cratering explosions have arisen widespread concern for a long time.
Actually, bulging and cratering explosions are more complex subjects which should take the influence of the free facet into consideration in dynamic models.

At present, the cratering mechanism of blasting can be divided into three kinds of effects: compressional stress wave effects, tensile reflected wave effects, and gas pressure effects. Since crushing and plastic deformation are omnipresent in the medium surrounding the explosive source, compressional stress wave effects predominate. After Hino [7] proposed the concept of blastability coefficient, reflected tensile wave effects have been widely accepted and increasingly investigated by other researchers. To clarify the role of gas pressure in the fragmentation of an underground blast, Kutter and Fairhurst [8] designed a system of model tests in which the combustion products were simulated by pressurised oil. They concluded that the gas pressure played an important role in blasting. Hagan agreed with this view and coined the term "pneumatic wedging" to describe previous observations $[9,10]$. Meanwhile, many other researchers, such as Dally et al. [11], Dally et al. [12], and 


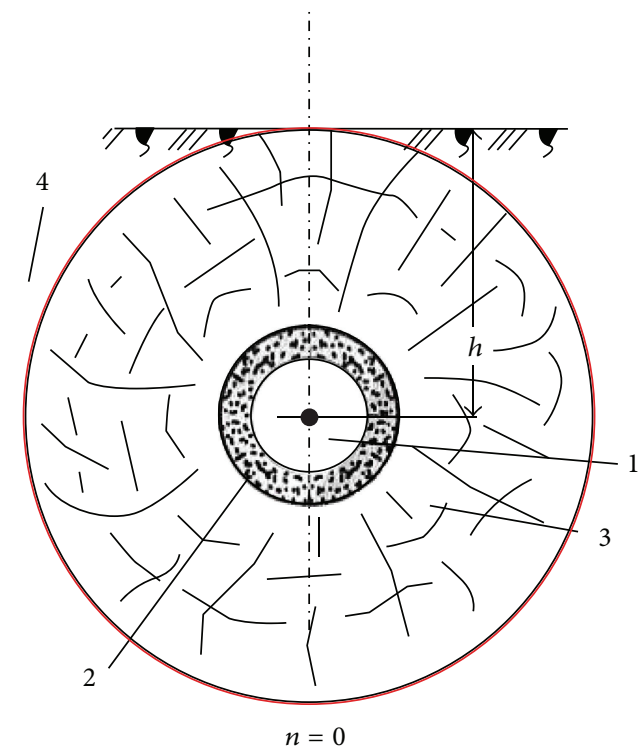

(a)

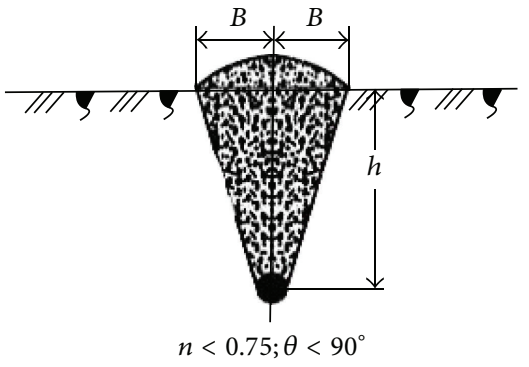

(b)

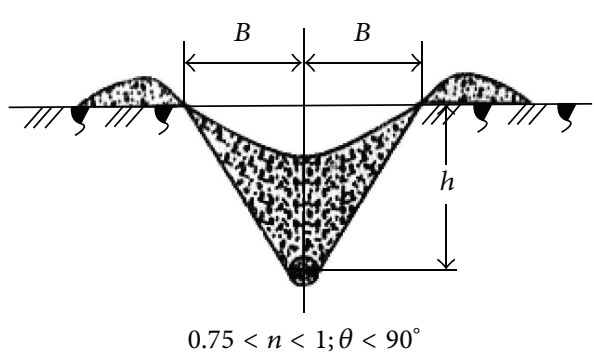

(c)

FIGURE 1: Underground explosions: (a) contained; (b) bulging; (c) cratering.

Harries [13], verified the reasonability of the aforementioned view in different ways. However, these research findings exhibit fundamental mechanisms of explosive cratering and cannot be used to solve most practical problems. Therefore, researchers have to formulate different hypotheses or conduct many tests to obtain any more practical computational formulae.

Nowadays, the widely used theories for calculating the explosive charge or the depth of burial of cratering explosions include Livingston's crater theory $[14,15]$, Boreskov's formula [16], Langefors' formula [17, 18], Vlasov's formula [19, 20], and Pokrovskii's formula [21, 22]. However, these theories cannot be used to calculate critical depth of burial of explosive charges to generate bulging and cratering in rock. Practically, it is important and pressing to investigate the mechanism or principle behind bulging explosions. A guide to bulging blasts in roads when earth-penetrating bombs are used on airport runway or highway pavements is needed.

As shown in Figure 1 [23], " $n$ " denotes throwing index, which is a ratio of the radius of the crater $(B)$ to the depth of burial of explosive $(h)$. For contained explosions, $n=0$, shown in Figure 1(a); for bulging explosions, $n$ $<0.75$, as shown in Figure 1(b); for cratering explosions, $n>0.75$, as shown in Figure 1(c). A critical depth of burial, which forms the dividing line between a contained explosion and a bulging explosion, can be called the first critical depth of burial (FCDOB). Similarly, a critical depth of burial which forms the dividing line between a bulging explosion and a cratering explosion can be called the second critical depth of burial (SCDOB). When the depth of burial is between these two critical depths, a bulging explosion tends to occur. This work focuses on two types of critical depth of burial, which can be used to predict the type of explosion and provide criteria for the occurrence of a bulging explosion.

\section{The Dynamic Model: An Introduction}

As is known, scabbing or perforation will occur when a target is subjected to blast loads [24, 25] (Figure 2). If the target is a semi-infinite medium and the explosive is detonated in the medium, scabbing or perforation will occur on the free facet and irreversible deformation happens near the blast hole; therefore, the progress is similar to that of a shallow buried explosion (Figure 3). Furthermore, the progress of a bulging explosion is similar to that of scabbing and the progress of a cratering explosion is similar to that of perforation. 


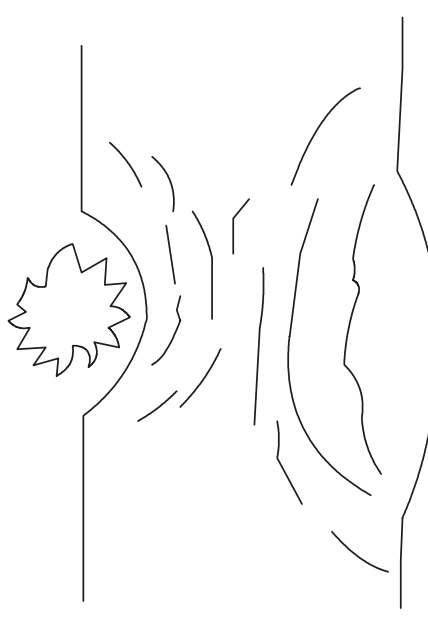

(a)

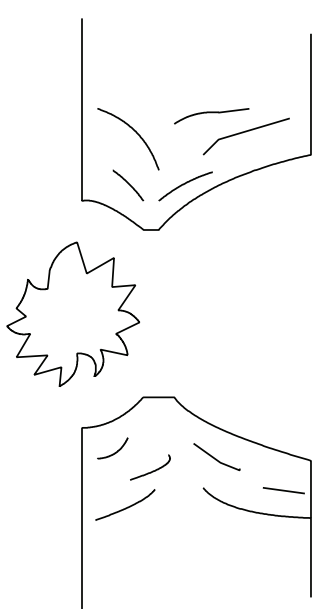

(b)

FIGURE 2: Local destructive effects of targets under blast loads: (a) scabbing; (b) perforation.

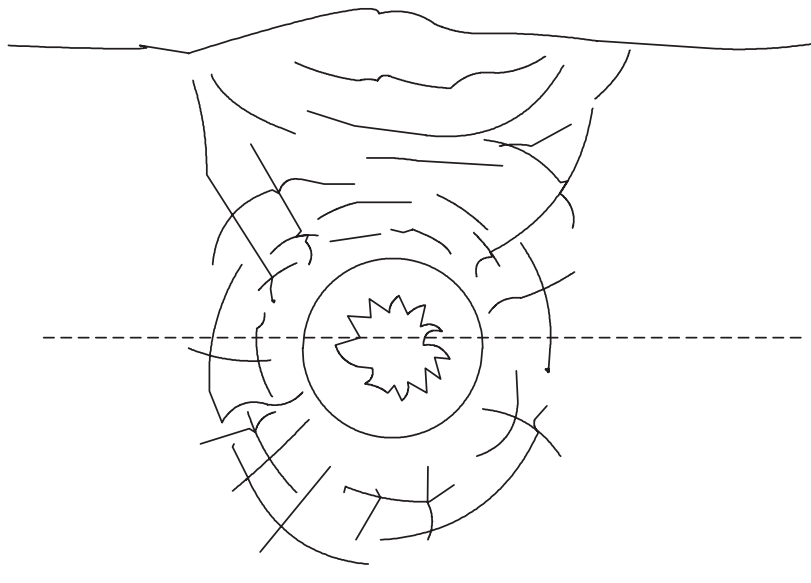

(a)

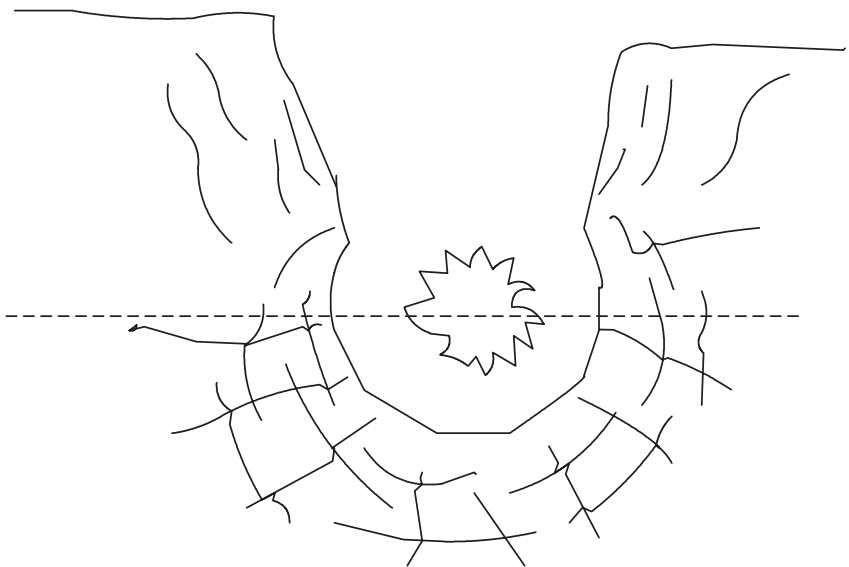

(b)

FIGURE 3: Shallow explosions: (a) bulging; (b) cratering.

Therefore, a method $[26,27]$ to calculate scabbing and perforation can be used to investigate the category of an underground explosion; however, the density of the medium, and the effective energy of the explosives, should be considered when establishing any dynamic model of a shallow buried explosion.

Before compressive waves reach the free surface, the processes in a shallow explosion are similar to those in a fully contained explosion. Hence, the initial conditions in a shallow-burial explosion are the same as the state parameters when coupled charges are detonated in an infinite media.

Figure 4 shows the proven results on the zoning of deformation and failure of rock under contained explosions [6]. There are four zones: (1) cavity; (2) crushed zone; (3) radial cracking zone; and (4) elastic zone. Besides, $R_{1}, R_{2}$, and $R_{3}$ are the cavity, crushed zone, and radial crack zone radii, respectively. However, all of them are posttest results and are measured after any explosion which cannot describe the dynamic processes inherent to explosive cavity expansion.

Regarding a thin layer near the cavity as an impact body [28], the problem of an explosion in rock can be transformed to a problem of impact between the impact body and the surrounding medium. Detonation products transmit effective initial mechanical energy to the surrounding medium through the impact body, which leads to irreversible deformation and movement of the medium. The impact body is a shell whose mass is constant and whose position moves outwards with the cavity-expansion velocity. As supposed by Forrestal and Tzou [29], when the cavity-expansion velocity 


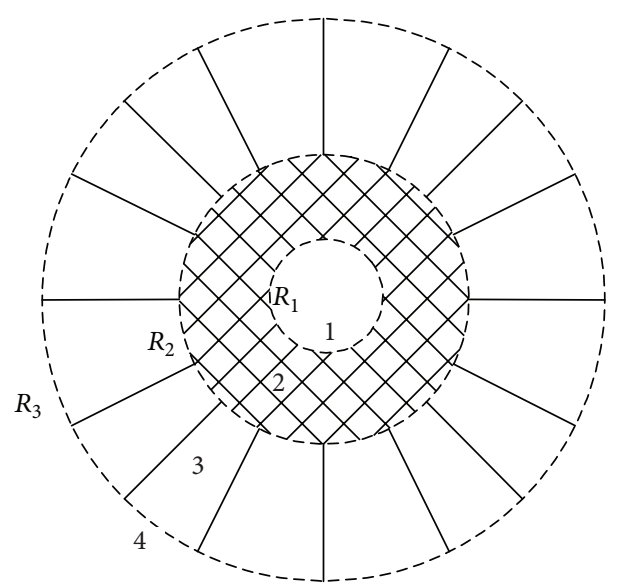

Figure 4: The zoning of deformation and failure of rock under a contained explosion.

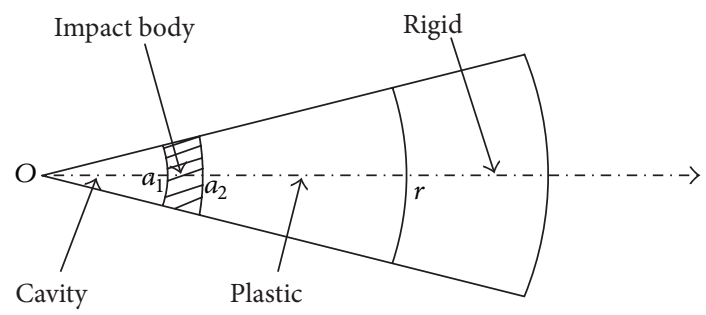

FIGURE 5: Outward motion of the impact body.

is large enough, the radially cracked region diminishes and the response can be depicted by an elastic-plastic model (Figure 5).

Radius $r$, as a varying displacement of the impact body, is the position of the contact surface between the impact body and the surrounding medium. Therefore, the initial conditions of the impact body can be written as

$$
\begin{aligned}
& \left.r\right|_{t=0}=a_{2}, \\
& \left.\dot{r}\right|_{t=0}=\bar{v}_{0} .
\end{aligned}
$$

According to the principle of the conservation of energy, the kinematic energy of the impact body is

$$
E_{k}=\frac{1}{2} M \bar{v}_{0}^{2}=\eta Q_{\nu} Q \delta
$$

where $\eta$ denotes a relative effective energy, $Q_{v}$ is the specific energy $(\mathrm{kcal} / \mathrm{kg}), Q$ is the mass of the charge $(\mathrm{kg})$, and $\delta=$ $4187(\mathrm{~J} / \mathrm{kcal})$ denotes the mechanical equivalent of heat.

The equation of motion of the impact body is

$$
M \ddot{r}=-P,
$$

where $M$ is the mass of the impact body, $r$ is the displacement of the impact body, and $P$ is the deformation resistance of the surrounding medium (material resistance).

The material resistance force can be acquired by seeking the limiting load. When the load reaches the limit for an

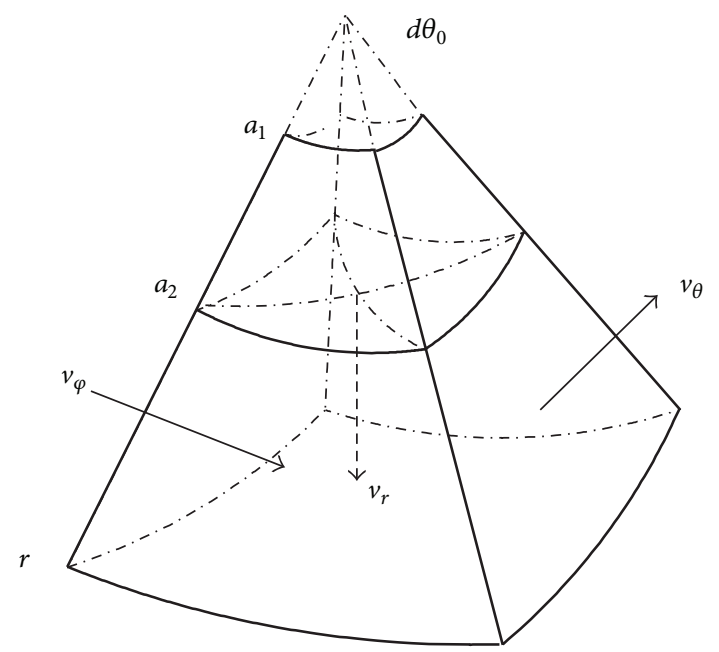

FIGURE 6: Velocity components in the velocity field of an infinite medium.

ideal plastic-rigid body (with a yield plateau), free plastic flow occurs. According to extremum principles and energy equilibrium conditions, the rate of work can be written as follows [27, 30, 31]:

$$
\int_{S} P_{n i}^{k} v_{i} d S=\int_{V} \sigma_{i j}^{k} \xi_{i j}^{k} d V+\sum_{k=1}^{N} \tau_{s} \int_{S_{\tau}}\left|\left[\nu_{\tau}^{k}\right]\right| d S_{\tau},
$$

where $S$ and $V$, respectively, are the area and the volume of the deformed body, $P_{i}^{k}$ are components of surface forces, $v_{i}$ are components of the possible kinematic velocity fields, $\xi_{i j}^{k}=$ $(1 / 2)\left(\partial v_{i}^{k} / \partial x_{j}+\partial v_{j}^{k} / \partial x_{i}\right)$ are components of strain rates, $\sigma_{i j}^{k}$ are components of the actual stress, $S_{\tau}$ are surfaces of velocity discontinuity, and $\left[v_{\tau}^{k}\right]$ denotes the jump $v_{\tau+}^{k}-v_{\tau-}^{k}$ on $S_{\tau}$.

By virtue of the Saint Venant-von Mises equation [31], it is found that the vectors $\sigma_{i j}^{k}$ and $\xi_{i j}^{k}$ are parallel and the quantity $\sigma_{i j}^{k} \xi_{i j}^{k}$, being the scalar product of parallel vectors, will be equal to the product of their moduli; that is, $\sigma_{i j}^{k} \xi_{i j}^{k}=\tau_{s} H^{k}$, where $\tau_{s}$ is the yield limit for shear and $H^{k}$ is the intensity of the shear strain-rate. Then (4) can be written as

$$
\int_{S} P_{i}^{k} \nu_{i} d S=\tau_{s} \int_{V} H^{k} d V+\sum_{k=1}^{N} \tau_{s} \int_{S_{\tau}}\left|\left[\nu_{\tau}^{k}\right]\right| d S_{\tau} .
$$

According to (4) or (5), the upper bound for the limit load of $P_{i}^{k}$ can be calculated from the possible kinematic velocity field. Therefore, the problem becomes one of how to accurately establish the possible kinematic velocity field which is based on experimental data.

\section{The Limiting Material Resistance Load}

3.1. Material Resistance to an Explosion in an Infinite Medium. In a spherical coordinate system (Figure 6), a microelement measuring $d \theta_{0}$ in both $\varphi$ and $\theta$ directions is cut from the model of a contained explosion. The sketch of a microelement 
near the cavity depicts the velocity components in the infinite medium velocity field, where $v_{r}, v_{\varphi}$, and $v_{\theta}$ are components of the velocity in $r, \varphi$, and $\theta$ directions, respectively; $a_{2}$ is the initial outer radius of the impact body, and $r$ is the new outer radius of the impact body which forms the contact surface at some point. From $a_{2}$ to $r$, the motion of the impact body is resisted by the surrounding medium which is in a plastic state. Then it is essential to find the material resistance. The limiting material resistance can be calculated as follows:

(1) Considering the symmetry of the velocity field, $v_{\theta}=$ $v_{\varphi}$. According to the law of conservation of mass, that is, $\bar{v}_{0} \cdot 4 \pi a_{2}^{2}=v_{r} \cdot 4 \pi r^{2}$, the radial velocity of the medium in the plastic zone is $v_{r}=a_{2}^{2} / r^{2} \bar{v}_{0}$. According to the incompressibility condition, $\partial v_{r} / \partial r+2\left(v_{r} / r\right)+$ $\partial v_{\varphi} / r \partial \varphi+\partial v_{\theta} / r \partial \theta=0$, and then $v_{\varphi}=v_{\theta}=0$.

(2) Since the velocity field is known, it is easy to obtain the strain-rate components:

$$
\begin{aligned}
& \xi_{r}=\frac{\partial v_{r}}{\partial r}=-2 \frac{a_{2}^{2}}{r^{3}} \bar{v}_{0}, \\
& \xi_{\theta}=\xi_{\varphi}=\frac{v_{r}}{r}=\frac{a_{2}^{2}}{r^{3}} \bar{v}_{0} .
\end{aligned}
$$

(3) The intensity of the shear strain-rate is

$$
H=\sqrt{2\left(\xi_{r}^{2}+\xi_{\theta}^{2}+\xi_{\varphi}^{2}\right)}=2 \sqrt{3} \frac{a_{2}^{2}}{r^{3}} \bar{v}_{0} .
$$

(4) Substitute the physical and geometric quantities into (5), manipulate algebraically, and then the limiting material resistance to an explosion in an infinite medium is

$$
\frac{P^{k}}{\tau_{s}}=2 \sqrt{3} \ln \left(\frac{r}{a_{2}}\right) .
$$

Using (8), material resistance that resists the motion of the impact body generated by the explosion in an infinite medium is calculated. It is a function of the yield limit for shear, the initial position of the contact surface, and the new position of the contact surface at some point.

\subsection{Material Resistance to Explosions in a Semi-Infinite} Medium. For explosions in a semi-infinite medium, it is essential to consider the effect of the free surface. There are two possible kinematic velocity fields: the first velocity field is a single-variable field where the radial velocity is related to the radial coordinate. It describes the motion when the free surface has been detected by the shock wave before the formation of a perforation body. However, the second velocity field is a constant field where the radial velocity remains constant over the whole deformation zone. It describes the motion after the formation of the perforation body which moves as one mass.

A funnel-shaped plastic region between the impact body and the free surface will be formed when the explosive is

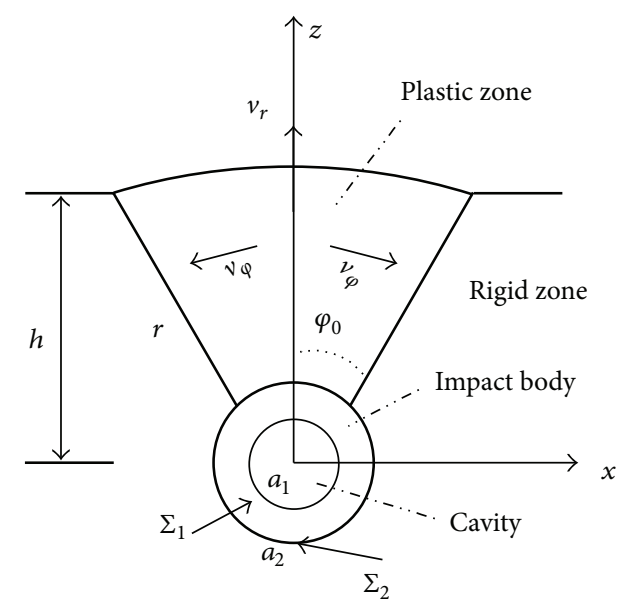

FIGURE 7: The first velocity field of explosions in a semi-infinite medium.

not buried deeply. In the funnel range, large displacements will occur as the medium is in an ideal plastic state; but, outside the funnel range, the deformation is restricted since the medium is seen as a rigid body. As shown in Figure $7, h$ is the depth of burial of the explosive; $a_{1}, a_{2}$ are, respectively, the initial inner radius and outer radius of the impact body; and $\varphi_{0}$ is the interfacial angle between the plastic zone and the rigid zone.

In the first velocity field, the plastic zone is the cone-shape region shown in Figure 7 whose cone angle is $\varphi_{0}$ and whose height ranges from $a_{2}$ to $h$. The initial zone of the impact body ranges from the internal surface $\Sigma_{1}$ to the external surface $\Sigma_{2}$. The area of surface $\Sigma_{1}$ cut by the cone is $S_{1}=2 \pi a_{1}^{2}\left(1-\cos \varphi_{0}\right)$; the volume of the cone inside surface $\Sigma_{1}$ is $V_{1}=2 / 3 \pi a_{1}^{3}(1-$ $\left.\cos \varphi_{0}\right)$; likewise, the area of surface $\Sigma_{2}$ cut by the cone, and the volume of the cone inside surface $\Sigma_{2}$, can be found.

Assuming that $v_{r}, v_{\varphi}$, and $v_{\theta}$ are velocity components in the $r, \varphi$, and $\theta$ directions (Figure 6), the limiting material resistance is calculated as follows:

(1) The initial velocity of the impact body is such that its average velocity is $\left.v_{r}\right|_{r=a_{2}}=\bar{v}_{0}$. According to the law of conservation of mass, the radial velocity is $v_{r}=\left(a_{2}^{2} / r^{2}\right) \bar{v}_{0}$. Considering the symmetry of the velocity field and the incompressibility condition, the compatible equations of this velocity field are $\partial v_{r} / \partial r+$ $2\left(v_{r} / r\right)+\partial v_{\varphi} / r \partial \varphi+\partial v_{\theta} / r \partial \theta=0$ and $v_{\theta}=0$. Combined with the condition $v_{r}=\left(a_{2}^{2} / r^{2}\right) \bar{v}_{0}, v_{\varphi}=0$.

(2) The strain-rate components are

$$
\begin{aligned}
& \xi_{r}=\frac{\partial v_{r}}{\partial r}=-2 \frac{a_{2}^{2}}{r^{3}} \bar{v}_{0}, \\
& \xi_{\theta}=\xi_{\varphi}=\frac{v_{r}}{r}=\frac{a_{2}^{2}}{r^{3}} \bar{v}_{0} .
\end{aligned}
$$

(3) The intensity of the shear strain-rate is

$$
H=\sqrt{2\left(\xi_{r}^{2}+\xi_{\theta}^{2}+\xi_{\varphi}^{2}\right)}=2 \sqrt{3} \frac{a_{2}^{2}}{r^{3}} \bar{v}_{0} .
$$




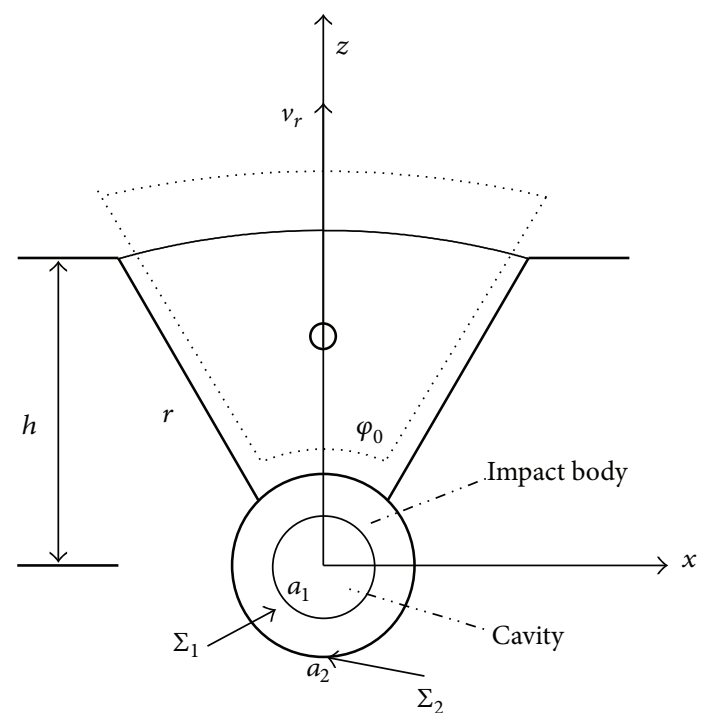

Figure 8: The second velocity field of an explosion in a finitethickness-medium.

(4) According to (5), the limiting material resistance to an explosion in a semi-infinite medium is

$$
\frac{P^{k}}{\tau_{s}}=\left(2 \sqrt{3}+\frac{\sin \varphi_{0}}{\left(1-\cos \varphi_{0}\right)}\right) \ln \left(\frac{h}{a_{2}}\right) .
$$

Equation (11) is solved at a certain time, at which the material resistance corresponds to the initial position of the contact surface. However, during the whole explosion process, the impact body undergoes outward movement and the position of the contact surface develops accordingly. It can be assumed that the position of the contact surface is $r$ at some point in the dynamic process, which is also the new outer radius of the impact body. Therefore, at some point, the limiting material resistance to explosions in a semi-infinite medium is calculated as

$$
\frac{P^{k}}{\tau_{s}}=\left(2 \sqrt{3}+\frac{\sin \varphi_{0}}{\left(1-\cos \varphi_{0}\right)}\right) \ln \left(\frac{h}{r}\right) .
$$

However, in the second velocity field (Figure 8), the cone-shape region undergoes rigid body motion. The velocity within the whole deformation zone remains unchanged, which indicates that the rigid block is pressed out from the medium.

Initially, the velocities of the rigid block and the impact body are different; therefore there remains a resistance to the effects of the explosion. As the motion develops, the rigid block and the impact share the same velocity; the resistance to explosion therefore becomes zero. This physical process characterises the block motion after the formation of a perforation.
After mathematical manipulation, the limiting material resistance to an explosion can be written in the similar form to that of (11):

$$
\frac{P^{k}}{\tau_{s}}=\frac{\tan \varphi_{0}}{2\left(1-\cos \varphi_{0}\right)}\left(\left(\frac{h}{a_{2}}\right)^{2}-1\right) .
$$

Likewise, at some point, the limiting material resistance to explosions in a semi-infinite medium is

$$
\frac{P^{k}}{\tau_{s}}=\frac{\tan \varphi_{0}}{2\left(1-\cos \varphi_{0}\right)}\left(\left(\frac{h}{r}\right)^{2}-1\right) .
$$

\section{Calculation Method: Critical Depth of Burial}

4.1. The Nondimensional Material Resistance. According to the literatures $[1,4,32-34]$, the cone angle of a standard blasting crater is $\pi / 4$. Therefore, it is assumed that the interfacial angle $\varphi_{0}=\pi / 4$. If the position of the contact surface $r$ is known, the material resistance in three velocity fields can be, respectively, acquired through (8), (12), and (14). Consequently, the relationships between material resistance and the contact surface position can be acquired. These are known as material resistance curves.

Equations (8), (12), and (14) can be transformed to nondimensional forms. Assuming $a_{0}$ is the radius of the explosive charge, for coupled charges, it is also the radius of the blast hole. Then the depth of burial $h=\tilde{h} \cdot a_{0}$, the position of contact surface $r=\tilde{r} \cdot a_{0}$, and the initial external radius of the impact body is $a_{2}=\tilde{a}_{2} \cdot a_{0}$.

From (8), the dimensionless material resistance in the velocity field in an infinite medium is

$$
\frac{P^{k}}{\tau_{s}}=2 \sqrt{3} \ln \left(\frac{r}{a_{2}}\right)=2 \sqrt{3} \ln \left(\frac{\tilde{r}}{\tilde{a}_{2}}\right) .
$$

From (12), the dimensionless material resistance in the first velocity field of a semi-infinite medium is written as

$$
\frac{P^{k}}{\tau_{s}}=5.88(\ln (\widetilde{h})-\ln (\widetilde{r})) .
$$

From (14), the dimensionless material resistance in the second velocity field of a semi-infinite medium is

$$
\frac{P^{k}}{\tau_{s}}=1.71\left(\left(\frac{\tilde{h}}{\tilde{r}}\right)^{2}-1\right) .
$$

Under the assumption that $h=10 a_{0}$, there are three function curves $P^{k} / \tau_{s} \sim \widetilde{r}$ (Figure 9): curve 1 is the material resistance curve in the first velocity field of a semi-infinite medium, curve 2 is the material resistance curve in the second velocity field of a semi-infinite medium, and curve 3 demonstrates the material resistance in the velocity field of an infinite medium. In Figure 9, curves 1 and 3 intersect at $A$, at which point the impact body begins to perceive the presence of the free surface when the position of the contact surface 


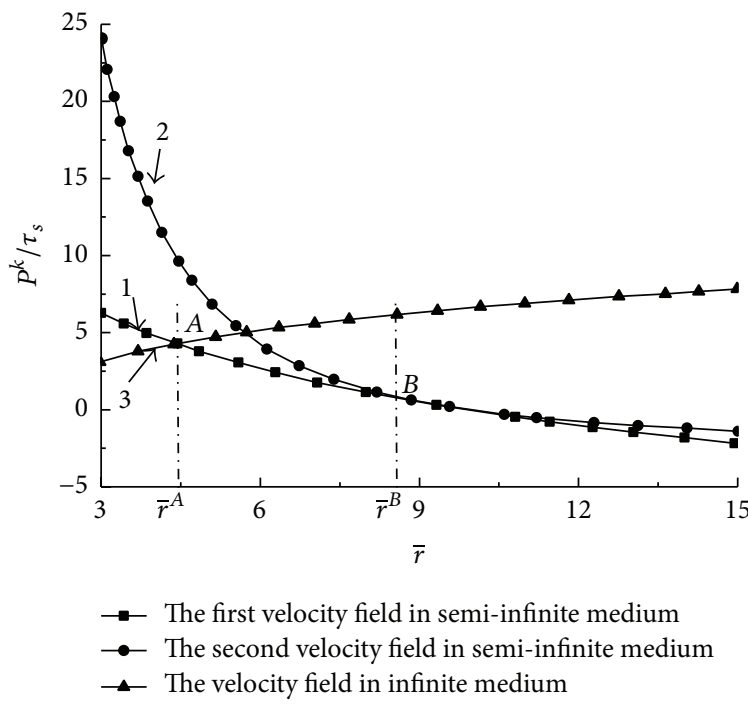

FIGURE 9: Material resistance curves.

reaches $\bar{r}^{A}$. Besides, curves 1 and 2 intersect at $B$, which indicates that the impact body pushes the mass block out of the surface when the position of the contact surface reaches $\bar{r}^{B}$. In the whole physical process, the material resistance is the minimum value among those on the three material resistance curves. To the left-hand side of $A$, the material resistance is given by curve 3 ; to the right-hand side of $A$, the material resistance is given by curve 1 .

Since $P^{k}$ is the force per unit area, the total force on the whole contact surface is

$$
P=P^{k} \cdot S_{r}
$$

where $S_{r}$ is the area of the contact surface, in an infinite medium, $S_{r}=4 \pi r^{2}$, and, in a semi-infinite medium, $S_{r}=$ $2 \pi r^{2}\left(1-\cos \varphi_{0}\right)$.

4.2. The First Critical Depth of Burial. According to (15) and (16), the total material resistance in an infinite medium $P=$ $P^{k} \cdot 4 \pi a_{0}^{2} \widetilde{r}^{2}$. Therefore, based on (3), the equation of motion in an infinite medium is

$$
\ddot{\widetilde{r}}=-\frac{8 \sqrt{3} \pi \tau_{s} a_{0}}{M} \ln \left(\frac{\tilde{r}}{\widetilde{a}_{2}}\right) \widetilde{r}^{2} .
$$

The initial conditions are calculated as

$$
\begin{aligned}
& \left.\tilde{r}\right|_{t=0}=\widetilde{a}_{2}, \\
& \left.\dot{\tilde{r}}\right|_{t=0}=\frac{v_{0}}{a_{0}} .
\end{aligned}
$$

Denoting $8 \sqrt{3} \pi \tau_{s} a_{0} / M$ by $\omega_{1}$, the equation of motion may be transformed to

$$
\ddot{\widetilde{r}}=-\omega_{1} \ln \left(\frac{\tilde{r}}{\widetilde{a}_{2}}\right) \widetilde{r}^{2} .
$$

As the differential equation cannot be solved directly, its transformed form is

$$
-\widetilde{v} \frac{d \widetilde{v}}{d \widetilde{r}}=\omega_{1} \ln \left(\frac{\widetilde{r}}{\tilde{a}_{2}}\right) \widetilde{r}^{2} .
$$

After separation of variables and integrating,

$$
\frac{1}{2}\left(\frac{v_{0}}{a_{0}}\right)^{2}=\frac{1}{3} \omega_{1} \ln \left(\frac{\tilde{r}}{\tilde{a}_{2}}\right) \tilde{r}^{3}-\frac{1}{9} \omega_{1} \widetilde{r}^{3}+\frac{1}{9} \omega_{1} \widetilde{a}_{2}^{3} .
$$

After simplifying,

$$
3 \ln \left(\frac{\tilde{r}}{\tilde{a}_{2}}\right) \widetilde{r}^{3}-\widetilde{r}^{3}+\widetilde{a}_{2}^{3}-\frac{4.5}{\omega_{1}}\left(\frac{v_{0}}{a_{0}}\right)^{2}=0 .
$$

Denoting $\left(4.5 / \omega_{1}\right)\left(v_{0} / a_{0}\right)^{2}$ by $\xi$, a dimensionless impact factor, by solving (24), we obtain

$$
\tilde{r}=\tilde{a}_{2} \cdot \exp \left[\frac{1}{3} W\left(-\frac{\tilde{a}_{2}^{3}-\xi}{\tilde{a}_{2}^{3} \cdot e}\right)+\frac{1}{3}\right],
$$

where $W(x)$ represents the Lambert $W$-function, and when the independent variable $x>-e^{-1}$, the function increases progressively, and the function result is a real single value.

Solving simultaneous equations (15) and (16) gives the medium thickness corresponding to point $A$ in Figure 9. This is also the depth of burial of the explosive and can be written as

$$
\widetilde{h}=\exp \left[-0.59 \ln \left(\widetilde{a}_{2}\right)+1.59 \ln (\widetilde{r})\right] .
$$

By substituting (25) into (26), the minimum depth of burial of explosive required to prevent scabbing becomes

$$
\widetilde{h}=\tilde{a}_{2} \cdot \exp \left[1.59\left(\frac{1}{3} W\left(-\frac{\tilde{a}_{2}^{3}-\xi}{\tilde{a}_{2}^{3} \cdot e}\right)+\frac{1}{3}\right)\right] .
$$

From (2), the form of the dimensionless impact factor becomes

$$
\xi=\frac{3}{2 \sqrt{3}} \frac{\eta Q_{\nu} \delta \rho_{0}}{\tau_{s}} .
$$

The dimensionless impact factor can also be expressed as follows:

$$
\xi=\frac{3}{2 \sqrt{3}} \frac{\eta Q_{v} \delta \rho_{0}}{\tau_{s}}=\lambda \eta\left(\frac{\rho_{0}}{\rho}\right)\left(\frac{\sigma_{d}}{\tau_{s}}\right)\left(\frac{Q_{v} \delta}{C_{p} v_{m}}\right) .
$$

It is a dimensionless combination of multiparameters, which characterises the relationships between the explosive density $\left(\rho_{0}\right)$, specific energy $\left(Q_{v}\right)$, the effective energy ratio $(\eta)$, medium density $(\rho)$, yield strength $\left(\tau_{s}\right)$, peak pressure $\left(\sigma_{d}\right)$, plastic wave velocity $\left(C_{p}\right)$, and maximum particle speed $\left(v_{m}\right)$.

The initial impact body is a thin shell around the cavity, and its outer radius $a_{2}=a_{0}(1+\varepsilon)$, where $\varepsilon \ll 1$ denotes the thickness of the shell. Therefore, $\widetilde{a}_{2}$, the dimensionless 
outer radius of the initial impact body, is $1+\varepsilon$, and it is approximately equal to 1 . Consequently, (27) becomes

$$
\tilde{h}=\exp \left[1.59\left(\frac{1}{3} W\left(-\frac{1-\xi}{e}\right)+\frac{1}{3}\right)\right]=f(\xi) .
$$

When using a coupled charge, $a_{0}$ is the radius of the explosive charge, and it is also the radius of the blast hole. Since the explosive density is assumed to be $\rho_{0}$, the mass of charge $Q=(4 / 3) \pi \rho_{0} a_{0}^{3}$. Then the relationship between the depth of burial and the mass of the charge is

$$
h=\tilde{h} \cdot a_{0}=\tilde{h} \cdot \sqrt[3]{\frac{Q}{4 \pi \rho_{0} / 3}} .
$$

Therefore

$$
\frac{h}{Q^{1 / 3}}=\frac{\tilde{h}}{\left(4 \pi \rho_{0} / 3\right)^{1 / 3}}=\frac{f(\xi)}{\left(4 \pi \rho_{0} / 3\right)^{1 / 3}} .
$$

Equation (32) denotes the scaled critical depth of burial for bulging, where the impact factor $\xi$ and the dimensionless critical depth of burial $\widetilde{h}$ are determined by (28) and (30). Equations (30) and (32) give the mass of the charge for bulging or scabbing as $Q=\left(4 \pi \rho_{0} / 3\right) /(f(\xi))^{3} \cdot h^{3}$. Therefore, $\left(4 \pi \rho_{0} / 3\right) /(f(\xi))^{3}$ is the functional expression of empirical coefficient $k_{s}$, which is the critical coefficient for scabbing.

4.3. The Second Critical Depth of Burial. To calculate the second critical depth of burial, this research uses the initial conditions consisting of the displacement and velocity corresponding to those at point $A$ in Figure 9. At point $A$, the impact body is affected by the existence of the free surface and the perforation begins to form. Then the material resistance (in motion) can be calculated from curve 1 between $A$ and $B$, which corresponds to the first velocity field in a semi-infinite medium. When the position of the contact surface $\tilde{r}$ reaches point $B$, the perforation ends and the cratering is complete. Therefore, the medium thickness $\widetilde{h}$ corresponding to point $B$ is the critical depth of burial for cratering.

According to (11) and (13), the material resistance in the first velocity field of a semi-infinite medium is $P=P^{k}$. $2 \pi a_{0}^{2} \widetilde{r}^{2}\left(1-\cos \varphi_{0}\right)$, and the mass of the impact body is $M=$ $(2 \pi / 3)\left(1-\cos \varphi_{0}\right)\left(\widetilde{r}_{a}^{3}-1\right) a_{0}^{3} \rho$; consequently, the equation of motion of the perforation is transformed to

$$
\ddot{\vec{r}}=-\frac{17.64 \tau_{s}}{\rho\left(\widetilde{r}_{a}^{3}-1\right) a_{0}^{2}} \ln \left(\frac{\widetilde{h}}{\tilde{r}}\right) \widetilde{r}^{2} .
$$

Combining the initial conditions $\left.\widetilde{r}\right|_{t=0}=\widetilde{r}_{a}$ and $\left.\dot{\tilde{r}}\right|_{t=0}=$ $v_{A} / a_{0}$ and denoting $17.64 \tau_{s} / \rho\left(\widetilde{r}_{a}^{3}-1\right) a_{0}^{2}$ by $\omega_{2}$, the equations of motion are transformed to

$$
\ddot{\widetilde{r}}=-\omega_{2} \ln \left(\frac{\tilde{h}}{\widetilde{r}}\right) \tilde{r}^{2} .
$$

This differential equation cannot be solved directly, and its transformed form is

$$
-\widetilde{v} \frac{d \widetilde{v}}{d \widetilde{r}}=\omega_{2} \ln \left(\frac{\tilde{h}}{\widetilde{r}}\right) \widetilde{r}^{2} .
$$

After separation of the variables and integration,

$$
\begin{aligned}
\frac{1}{2}\left(\frac{v_{A}}{a_{0}}\right)^{2}= & \frac{1}{3} \omega_{2} \ln \left(\frac{\widetilde{h}}{\widetilde{r}}\right) \tilde{r}^{3}+\frac{1}{9} \omega_{2} \widetilde{r}^{3} \\
& -\frac{1}{3} \omega_{2} \ln \left(\frac{\tilde{h}}{\widetilde{r}_{a}}\right) \widetilde{r}_{a}^{3}-\frac{1}{9} \omega_{2} \widetilde{r}_{a}^{3} .
\end{aligned}
$$

The initial conditions of perforation were given by (21), so

$$
\begin{aligned}
\frac{1}{2}\left(\frac{v_{0}}{a_{0}}\right)^{2}-\frac{1}{2}\left(\frac{v_{A}}{a_{0}}\right)^{2}= & \frac{1}{3} \omega_{1} \ln \left(\frac{\tilde{r}_{a}}{\widetilde{a}_{2}}\right) \widetilde{r}_{a}^{3}-\frac{1}{9} \omega_{1} \tilde{r}_{a}^{3} \\
& +\frac{1}{9} \omega_{1} \tilde{a}_{2}^{3} .
\end{aligned}
$$

According to the initial conditions on the impact body, the aforementioned equation may be transformed to

$$
\begin{aligned}
\frac{1}{2}\left(\frac{v_{0}}{a_{0}}\right)^{2}-\frac{1}{2}\left(\frac{v_{A}}{a_{0}}\right)^{2}= & \frac{1}{3} \omega_{1} \ln \left(\widetilde{r}_{a}\right) \widetilde{r}_{a}^{3}-\frac{1}{9} \omega_{1} \widetilde{r}_{a}^{3} \\
& +\frac{1}{9} \omega_{1} .
\end{aligned}
$$

Solving (36) and (38) simultaneously gives, after algebraic manipulation.

$$
\begin{aligned}
& \left(3 \ln \left(\frac{\tilde{h}}{\widetilde{r}_{b}}\right) \tilde{r}_{b}^{3}+\widetilde{r}_{b}^{3}-3 \ln \left(\frac{\tilde{h}}{\widetilde{r}_{a}}\right) \tilde{r}_{a}^{3}-\widetilde{r}_{a}^{3}\right) \cdot \frac{\omega_{2}}{\omega_{1}}-\xi_{2} \\
& =0 \\
& \xi_{2}=\frac{4.5}{\omega_{1}}\left(\frac{v_{0}}{a_{0}}\right)^{2}-3 \ln \left(\widetilde{r}_{a}\right) \tilde{r}_{a}^{3}+\tilde{r}_{a}^{3}-1 \\
& =\xi-3 \ln \left(\tilde{r}_{a}\right) \tilde{r}_{a}^{3}+\tilde{r}_{a}^{3}-1,
\end{aligned}
$$

where $\omega_{1}=8 \sqrt{3} \pi \tau_{s} a_{0} / M=8 \sqrt{3} \pi \tau_{s} /\left(4 \pi / 3 \cdot \rho\left(\widetilde{r}^{3}-1\right) a_{0}^{2}\right)$ and $\omega_{2}=17.64 \tau_{s} / \rho\left(\widetilde{r}_{a}^{3}-1\right) a_{0}^{2}$. Since $\tilde{r}=\widetilde{r}_{a}$ as an initial condition,

$$
\frac{\omega_{2}}{\omega_{1}}=1.697
$$

From point $A$ (Figure 9), the relationship between the position of the contact surface and the medium thickness can be written as:

$$
\left.\widetilde{r}\right|_{t=0}=\widetilde{r}_{a}=\widetilde{h}^{0.63} .
$$

From point $B$ (Figure 9), the relationship between the position of the contact surface and the medium thickness can be written as

$$
\left.\widetilde{r}\right|_{t=t^{*}}=\widetilde{r}_{b}=\frac{\widetilde{h}}{1.65} .
$$

Combining (39) with (42), which include equations of motion, boundary conditions, and initial conditions, an equation for the critical depth of burial for cratering is obtained:

$$
0.945 \widetilde{h}^{3}+0.006 \ln (\widetilde{h}) \widetilde{h}^{1.89}-2.697 \widetilde{h}^{1.89}-\xi+1=0,
$$




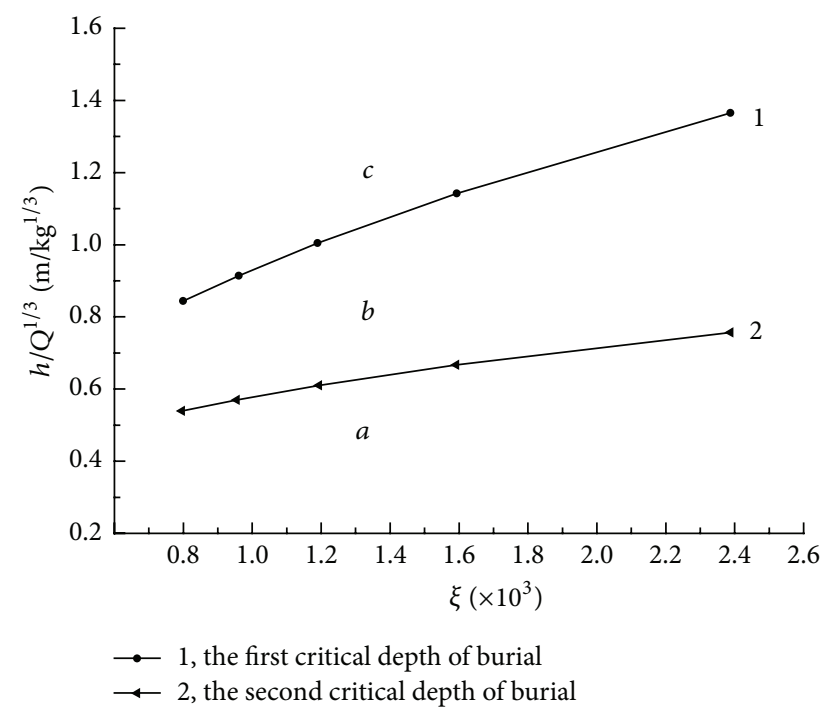

FIgURE 10: Calculated critical depths of burial.

where $\widetilde{h}=g(\xi)$ denotes the critical depth of burial for cratering and $\xi$ denotes the dimensionless impact factor. However, (43) cannot be solved by using an analytic method, and there is no choice but to pursue an approximate solution by numerical method. Then, substituting the solution into (31) and manipulating algebraically, the scaled critical depth of burial for cratering can be written in the same form as (32). Similarly, from (32) and (43), the mass of the charge required for cratering or perforation is given by $Q=\left(4 \pi \rho_{0} / 3\right) /(g(\xi))^{3}$. $h^{3}$. Therefore, $\left(4 \pi \rho_{0} / 3\right) /(g(\xi))^{3}$ is the functional expression of empirical coefficient, and $k_{p}$ is the critical coefficient for perforation.

\section{Calculation of Critical Depths of Burial}

Assuming that the key variables [35] are known, they consist of explosive density $\rho_{0}=1630 \mathrm{~kg} / \mathrm{m}^{3}$, the effective energy ratio $\eta \approx 0.8$, specific energy $Q_{v}=1010 \mathrm{kcal} / \mathrm{kg}$, mechanical equivalent of heat $\delta=4187 \mathrm{~J} / \mathrm{kcal}$, medium density $\rho=$ $2600 \mathrm{~kg} / \mathrm{m}^{3}$, and material yield strength which ranges from $6 \mathrm{MPa}$ to $2 \mathrm{MPa}$.

According to (28), (30), (32), and (43), the scaled forms of the critical depth of burial curves $h / Q^{1 / 3} \sim \xi$ are illustrated in Figure 10.

In Figure 10, as the material yield strength decreases, the dimensionless impact factor $\xi$ increases, and the critical depth of burial $h / Q^{1 / 3}$ rises. It indicates that materials with higher yield strengths confer a greater blast-resistance and, therefore, have a smaller explosive critical depth of burial requirement to prevent bulging or cratering. These results are consistent with empirical conclusions. Some cases involving each critical depth of burial are summarised in Table 1.

From Table 1, the FCDOB ranges from $0.8 \mathrm{~m} / \mathrm{kg}^{1 / 3}$ to $1.4 \mathrm{~m} / \mathrm{kg}^{1 / 3}$, and the SCDOB ranges from $0.5 \mathrm{~m} / \mathrm{kg}^{1 / 3}$ to $0.7 \mathrm{~m} / \mathrm{kg}^{1 / 3}$. Actually, the scope of underground explosions can be empirically divided into three types, whose critical depths of burial are listed as follows [36, 37]: those whose FCDOB ranges from $1 \mathrm{~m} / \mathrm{kg}^{1 / 3}$ to $2 \mathrm{~m} / \mathrm{kg}^{1 / 3}$ and whose SCDOB ranges from $0.4 \mathrm{~m} / \mathrm{kg}^{1 / 3}$ to $0.6 \mathrm{~m} / \mathrm{kg}^{1 / 3}$. The critical depths of burial calculated by using the proposed theoretical method are only approximately equal to the empirical values found. In addition, two types of critical depth of burial have divided the scope of underground explosions into three regions- $a, b$, and $c$-which correspond to contained explosions, bulging explosions, and cratering explosions, respectively.

\section{Conclusion and Recommendations for Future Research}

This research transformed the problem of an explosion to one of an impact between a moving body and the surrounding medium. Then it derived the material resistance to explosive loading using limit load theorems and solved the equation of motion by combining the initial conditions and the boundary conditions. Finally, the research found two types of critical depth of burial for bulging and cratering and proved the correctness of the method through use of a case study. On the basis of the above calculations and analysis, the following was concluded:

(1) Two types of critical depth of burial are both positively related to the dimensionless impact factor. The dimensionless impact factor $\xi$ reflects the properties of the explosive performance and material strength, and it also presents the blasting ability of an explosive on its surrounding materials. The higher the explosive parameters, the more effective the explosive and the bigger the dimensionless impact factor; therefore, the critical depth of burial is greater. On the contrary, the higher the material yield strength, the stronger the material resistance to explosion and the smaller the dimensionless impact factor; therefore, the critical depth of burial is smaller.

(2) In the $h / Q^{1 / 3} \sim \xi$ plane, two critical depth of burial curves divide the plane into three regions corresponding to contained explosions, bulging explosions, and cratering explosions, respectively. Besides, the critical depths of burial calculated by the proposed theoretical method are approximately equal to the empirical results found.

(3) Based on the comparison with empirical results, the theoretical calculation method is verified to be reasonable. Besides, the theoretical method explains the physical essence of the empirical critical coefficients.

Since the critical depth of burial is a theoretical value, it is too difficult to implement an explosion which is located exactly on the critical depth of burial curve locus. At the same time, although there is much information about underground explosions, some key parameters, such as the effective energy ratio and material yield strength, are usually not given in most references. Therefore, it is difficult to verify the precision of the theoretical method. However, some verification tests, 
TABLE 1: Calculated results: two types of critical depth of burial.

\begin{tabular}{|c|c|c|c|c|c|}
\hline Dimensionless impact factor & 795.942 & 955.130 & 1193.913 & 1591.884 & 2387.825 \\
\hline${ }^{* 1} \mathrm{FCDOB}\left(\mathrm{m} / \mathrm{kg}^{1 / 3}\right)$ & 0.844 & 0.914 & 1.004 & 1.142 & 1.365 \\
\hline${ }^{* 2} \operatorname{SCDOB}\left(\mathrm{m} / \mathrm{kg}^{1 / 3}\right)$ & 0.540 & 0.570 & 0.610 & 0.667 & 0.757 \\
\hline${ }^{* 3} \mathrm{RCDOB}$ & 1.563 & 1.604 & 1.645 & 1.712 & 1.803 \\
\hline
\end{tabular}

${ }^{* 1}$ FCDOB denotes the "first critical depth of burial."

${ }^{* 2}$ SCDOB denotes the "second critical depth of burial."

${ }^{* 3} \mathrm{RCDOB}$ denotes the "ratio of first critical depth of burial to second critical depth of burial."

which are more targeted towards the proposed calculation method, are designed. Generation of more data and verification in support of the postulated accuracy of the method are recommended.

\section{Nomenclature}

$R_{1}$ : The radius of the cavity

$R_{2}$ : The radius of the crushed zone

$R_{3}$ : The radius of the radical crack zone

$r$ : The radius of the spherical shell

$a_{0}$ : The radius of the charge

$\rho_{0}$ : $\quad$ The density of charge

$h$ : The depth of burial of explosives

$B$ : $\quad$ The radius of crater on the surface

$n$ : $\quad$ The throwing index

$a_{1}$ : The initial radius of cavity

$a_{2}: \quad$ The initial radius of the spherical shell

$\bar{v}_{0}: \quad$ The initial velocity of the spherical shell

$\eta$ : $\quad$ A relative effective energy

$Q_{v}: \quad$ The specific energy of explosives

$Q: \quad$ The mass of the charge

$\delta: \quad$ The mechanical equivalent of heat

$E_{k}$ : The kinetic energy of the spherical shell

M: The mass of the spherical shell

$P$ : $\quad$ Material resistance

$S: \quad$ The area of the deformed body

$V: \quad$ The volume of the deformed body

$P_{i}^{k}: \quad$ Components of surface forces

$v_{i}$ : Components of the kinematic velocity fields

$\xi_{i j}^{k}: \quad$ Components of strain rates

$\sigma_{i j}^{k}: \quad$ Components of the actual stress

$\left[v_{\tau}^{k}\right]:$ The jump of velocity on surface $S_{\tau}$

$\tau_{s}: \quad$ The yield limit for shear

$H^{k}$ : The intensity of the shear strain-rate

$\widetilde{r}, \widetilde{h}, \widetilde{a}_{2}$ : The nondimensional forms of $r, h$, and $a_{2}$

$\xi$ : $\quad$ A dimensionless impact factor

$W(x)$ : The Lambert $W$-function

M: $\quad$ The mass of the spherical shell.

\section{Competing Interests}

The authors declare that there are no competing interests regarding the publication of this paper.

\section{Acknowledgments}

The authors acknowledge the financial support from the Programme for Changjiang Scholars and Innovative Research Teams in Universities (Grant no. IRT13071) and the Natural Science Foundation of China (Grant nos. 51308543 and 51308542).

\section{References}

[1] M. D. Nordyke, On Cratering: A Brief History, Analysis, and Theory of Cratering, Nuclear Explosions-Peaceful Applications, California University, Lawrence Radiation Lab, Livermore, Calif, USA, 1961.

[2] P. Chadwick, A. Kochs, and G. Hopkinson, Mechanics of Deep Underground Explosions, Philosophical Transactions of the Royal Society of London. Series A, Mathematical and Physical Sciences, Royal Society, London, UK, 1964.

[3] A. H. Hanukaliyev, Physical Processes on Rock Blasting, Metallurgical Industry Press, Moscow, Russia, 1974.

[4] K. R. Housen, R. M. Schmidt, and K. A. Holsapple, "Crater ejecta scaling laws: fundamental forms based on dimensional analysis," Journal of Geophysical Research: Solid Earth (19782012), vol. 88, pp. 2485-2499, 1983.

[5] W. L. Fourney, R. D. Dick, and K. R. Y. Simha, "Model study of crater blasting," Rock Mechanics and Rock Engineering, vol. 21, no. 3, pp. 183-205, 1988.

[6] Q. Qian, "Some advances in rock blasting dynamics," Chinese Journal of Rock Mechanics and Engineering, vol. 28, no. 10, pp. 1945-1968, 2009.

[7] K. Hino, "Fragmentation of rock through blasting and shock wave theory of blasting," in Proceedings of the 1st US Symposium on Rock Mechanics (USRMS '56), American Rock Mechanics Association, Golden, Colo, USA, April 1956.

[8] H. K. Kutter and C. Fairhurst, "On the fracture process in blasting," International Journal of Rock Mechanics and Mining Sciences, vol. 8, pp. 181-202, 1971.

[9] T. N. Hagan, "The influence of controllable blast parameters on fragmentation and mining costs," in Proceedings of the 1st International Symposium on Rock Fragmentation by Blasting, Lulea, Sweden, 1983.

[10] T. N. Hagan, "Rock breakage by explosives," Acta Astronautica, vol. 6, no. 3-4, pp. 329-340, 1979.

[11] J. W. Dally, W. L. Fourney, and D. C. Holloway, "Influence of containment of the bore hole pressures on explosive induced fracture," International Journal of Rock Mechanics and Mining Sciences and, vol. 12, no. 1, pp. 5-12, 1975.

[12] J. W. Dally, W. L. Fourney, and G. R. Irwin, "On the uniqueness of the stress intensity factor-crack velocity relationship," in 
Dynamic Fracture, pp. 33-42, Springer, Amsterdam, Netherlands, 1985.

[13] G. Harries, "Theory of blasting," in Drilling \& Blasting Technology, Australian Mineral Foundation, 1977.

[14] C. W. Livingston, "Explosions in snow," DTIC Document, 1968.

[15] C. W. Livingston, “Scaled charge depth," Google Patents, 1974.

[16] M. M. Boreskov, Experience in Mining Engineering, Department of Principalities Press, St. Petersburg, Russia, 1869 (Russian).

[17] U. Langefors and B. Kihlström, The Modern Technique of Rock Blasting, John Wiley \& Sons, New York, NY, USA, 1978.

[18] U. Langefors, "Bench blasting with an-explosives," Mining Research, pp. 249-271, 1962.

[19] O. E. Vlasov and S. A. Smirnov, Foundations of the Rock Blast, House of the USSR Academy of Sciences, 1962 (Russian).

[20] O. E. Vlasov, Fundamental Theory of Explosion, Military History Archive Press, Moscow, Russia, 1957 (Russian).

[21] G. I. Pokrovskii, "On the use of nuclear explosives for industrial purposes," Izvestiya Vysshikh Uchebnykh Zavedenii: Seriya Gornyi Zhurnal, vol. 5, pp. 29-32, 1956.

[22] G. I. Pokrovskii, Blasting, Nedra, Moscow, Russia, 1973.

[23] http://scholar.google.com.cn/.

[24] Q.-P. Zheng, Q.-H. Qian, Z.-S. Zhou, J.-H. Hu, and J.-X. $\mathrm{Xu}$, "Comparative analysis of scabbing thickness estimation of reinforced concrete structures," Engineering Mechanics, vol. 20, no. 3, pp. 47-53, 2003.

[25] K. Ohkubo, M. Beppu, T. Ohno, and K. Satoh, "Experimental study on the effectiveness of fiber sheet reinforcement on the explosive-resistant performance of concrete plates," International Journal of Impact Engineering, vol. 35, no. 12, pp. 17021708, 2008.

[26] V. M. Fomin, A. I. Gulidov, G. A. Sapozhnikov et al., High-Speed Interaction of Bodies, Siberian Branch of the Russian Academy of Sciences, Novosibirsk, Russia, 1999 (Russian).

[27] M.-Y. Wang, S.-L. Chen, and Y.-F. Pan, "Method of calculation for the penetration of a flat-nosed projectile in the rock (concrete)," Acta Armamentarii, vol. 26, no. 1, pp. 46-52, 2005.

[28] C. Z. Qi and Q. H. Qian, Basic Problems of Dynamic Deformation and Fracture of Rock Mass, Science Press, Beijing, China, 2009.

[29] M. J. Forrestal and D. Y. Tzou, "A spherical cavity-expansion penetration model for concrete targets," International Journal of Solids and Structures, vol. 34, no. 31-32, pp. 4127-4146, 1997.

[30] R. Hill, The Mathematical Theory of Plasticity, Oxford University Press, Oxford, UK, 1950.

[31] L. M. Kachanov, Foundations of the Theory of Plasticity, NorthHolland, Amsterdam, The Netherlands, 1971.

[32] L. P. Orlenko, Explosion Physics, Fizmatlit, Moscow, Russia, 2004.

[33] T.-Q. Ye, "Field experiment for blasting crater," Journal of China University of Mining and Technology, vol. 18, no. 2, pp. 224-228, 2008.

[34] Q. H. Qian and M. Y. Wang, Impact and Explosion Effects in Rock and Soil, National Defense Industry Press, Beijing, China, 2010.

[35] J. Henrych, The Dynamics of Explosion and Its Use, Elsevier Scientific Publishing Company, 1979.

[36] V. V. Adushkin and A. A. Spivak, Geomechanics of Large-Scale Explosions, Nedra, Moscow, Russia, 1993.

[37] V. V. Adushkin and A. A. Spivak, Underground Explosions, Sciences Press, 2007 (Russian). 


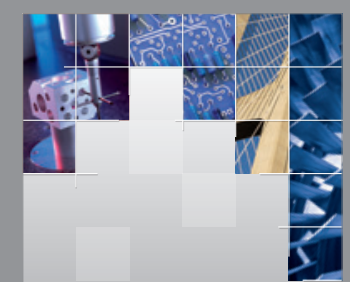

\section{Enfincering}
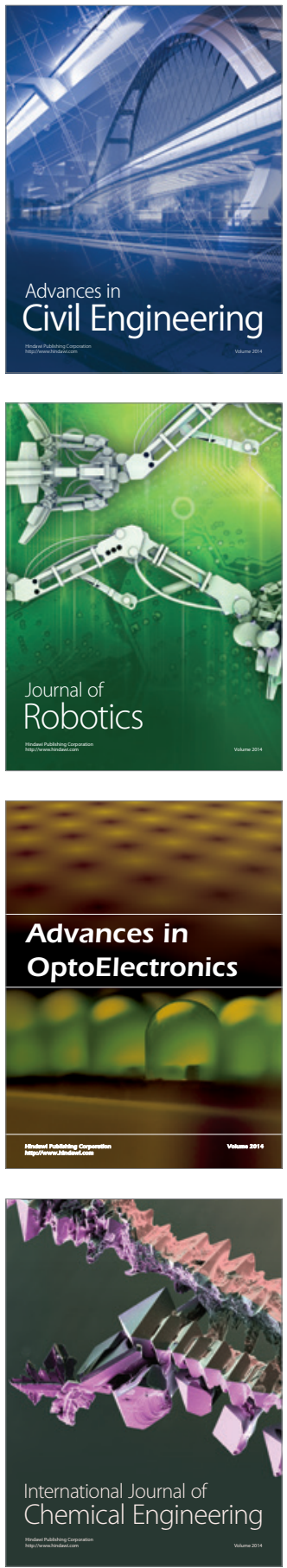

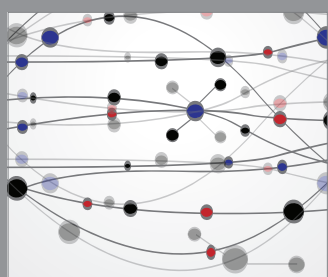

The Scientific World Journal

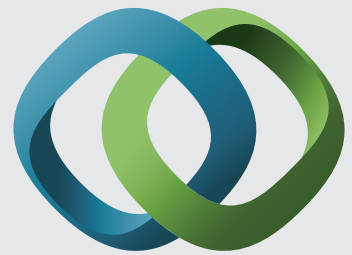

\section{Hindawi}

Submit your manuscripts at

http://www.hindawi.com
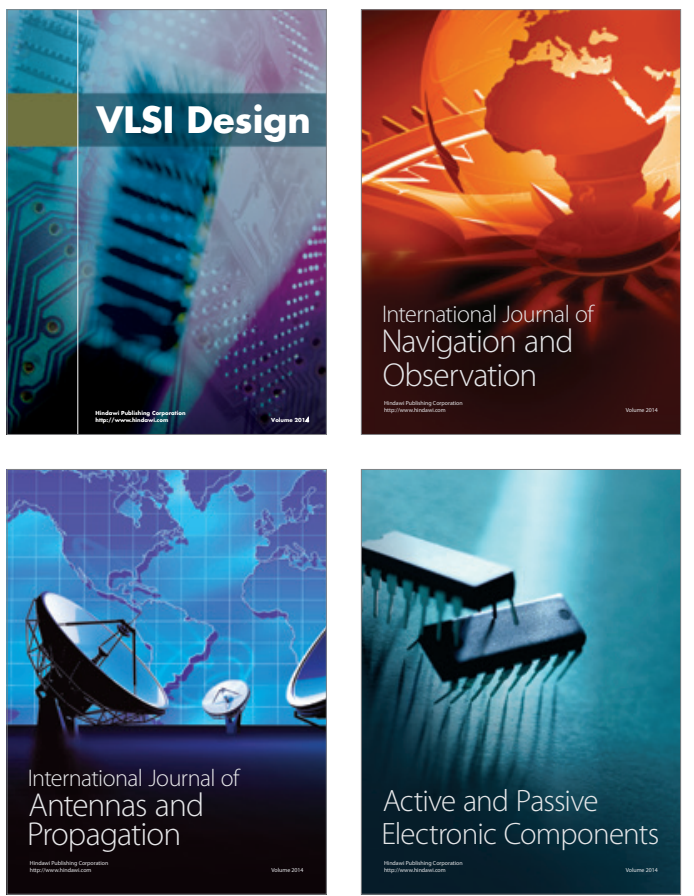
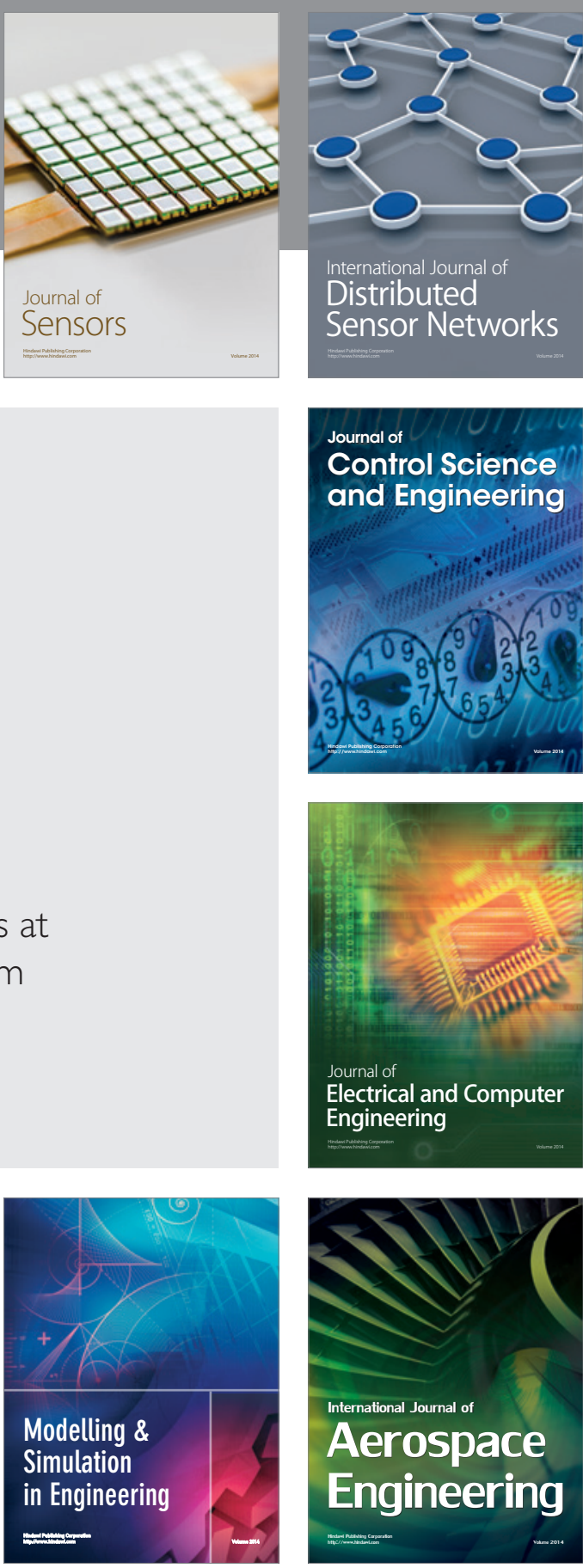

International Journal of

Distributed

Sensor Networks

Journal of

Control Science

and Engineering
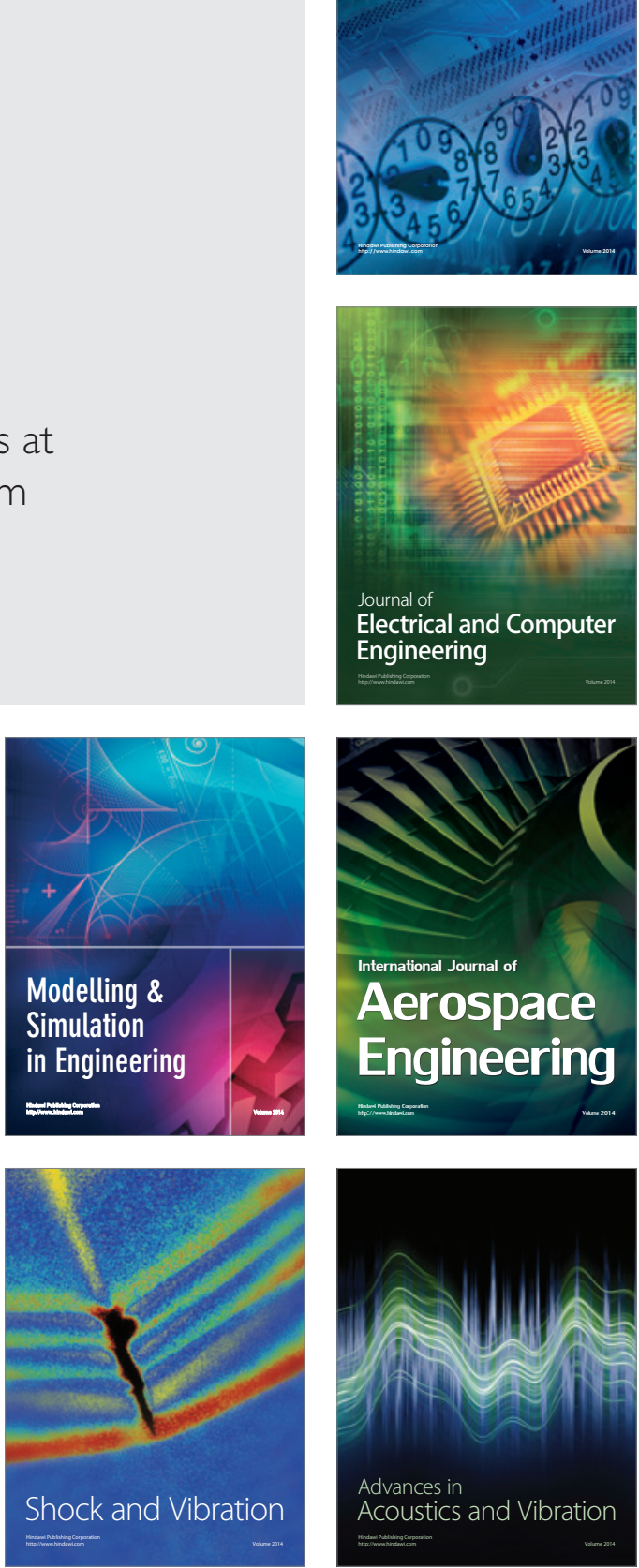